\title{
Four Point Implicit Methods for the Second Derivatives of the Solution of First Type Boundary Value Problem for One Dimensional Heat Equation
}

\author{
Suzan Cival Buranay ${ }^{1, *}$ and Lawrence Adedayo Farinola ${ }^{1}$ \\ ${ }^{1}$ Department of Mathematics, Faculty of Arts and Sciences, Eastern Mediterranean University, \\ Famagusta, North Cyprus, Via Mersin 10 Turkey
}

\begin{abstract}
We construct four-point implicit difference boundary value problem for the first derivative of the solution $u(x, t)$ of the first type boundary value problem for one dimensional heat equation with respect to the time variable $t$. Also, for the second derivatives of $u(x, t)$ special fourpoint implicit difference boundary value problems are proposed. It is assumed that the initial function belongs to the Hölder space $C^{8+\alpha}, 0<\alpha<1$, the heat source function given in the heat equation is from the Hölder space $C_{x, t}^{6+\alpha, 3+\frac{\alpha}{2}}$, the boundary functions are from $C^{4+\frac{\alpha}{2}}$, and between the initial and the boundary functions the conjugation conditions of orders $q=0,1,2,3,4$ are satisfied. We prove that the solution of the proposed difference schemes converge uniformly on the grids of the order $\mathrm{O}\left(h^{2}+\tau\right)$ (second order accurate in the spatial variable $x$ and first order accurate in time $t$ ) where, $h$ is the step size in $x$ and $\tau$ is the step size in time. Theoretical results are justified by numerical examples.
\end{abstract}

\section{Introduction}

Many practical heat conduction questions lead to problems not conveniently solvable by classical methods such as separation of variables techniques or the use of Green's functions. As a result approximate procedures like finite difference methods have been proposed, (see [1] and the references there in). On the other hand, besides the solution of the heat conduction problem it is very important to provide information about some physical phenomena related with the derivatives. Such as, there are a number of problems in heat conduction in which one material is transformed into another with generation or absorption of heat. Examples are the melting or freezing of a solid and the process of a temperaturedependent chemical reaction through a solid [2], [3]. In melting problems to calculate the

\footnotetext{
*Corresponding author: suzan.buranay@emu.edu.tr
} 
quantities as the rate of change in temperature $(\partial u / \partial t)$, the gradient of the rate of change in temperature $\left(\partial^{2} u / \partial t^{2}\right)$, the rate of heat flow into the solid $(\partial u / \partial x)$, the gradient of the rate of heat flow into the solid $\left(\partial^{2} u / \partial x^{2}\right)$ and the rate of melting $(\partial s / \partial t)$ where $x=s(t)$ is the thickness melted, $u(x, t)$ is the temperature of the solid are considerably important.

In this study we consider the first type boundary value problem for one dimensional heat equation of which the initial function belongs to $C^{8+\alpha} 0<\alpha<1$, the heat source function is from $C_{x, t}^{6+\alpha, 3+\frac{\alpha}{2}}$, the boundary functions are from $C^{4+\frac{\alpha}{2}}$, and between the initial and the boundary functions the conjugation conditions of orders $q=0,1,2,3,4$ are satisfied. Denoting the exact solution of this problem by $u(x, t)$, difference problems of four point implicit schemes approximating $\frac{\partial u}{\partial t}, \frac{\partial^{2} u}{\partial x^{2}}, \frac{\partial^{2} u}{\partial t^{2}}$ and $\frac{\partial^{2} u}{\partial x \partial t}$ are constructed. It is obtained that the solution of the constructed difference schemes converge uniformly to the exact values of $\frac{\partial u}{\partial t}, \frac{\partial^{2} u}{\partial x^{2}}, \frac{\partial^{2} u}{\partial t^{2}}$ and $\frac{\partial^{2} u}{\partial x \partial t}$ respectively, on the grids of order $O\left(h^{2}+\tau\right)$.

2 Implicit Four-Point Difference Approximation of $\frac{\partial u}{\partial t}, \frac{\partial^{2} u}{\partial x^{2}}, \frac{\partial^{2} u}{\partial t^{2}}$ and $\frac{\partial^{2} u}{\partial x \partial t}$

Let $\Omega=(0, b), \bar{\sigma}=(0, T)$, and $\bar{\Omega}, \bar{\sigma}_{T}$ be the closure of these sets respectively. Also denote by $Q_{T}=\{(x, t): 0<x<b, 0<t \leq T\}, \gamma_{1}=\left\{(0, t): t \in \bar{\sigma}_{T}\right\}, \gamma_{2}=\{(x, 0): x \in \bar{\Omega}\}$, $\gamma_{3}=\left\{(b, t): t \in \bar{\sigma}_{T}\right\}$. Let $\gamma=\bigcup_{i=1}^{3} \gamma_{i}$, represent the boundary of $Q_{T}$, and $\bar{Q}_{T}=Q_{T} \cup \gamma$.

Let $\mathrm{s}$ be a noninteger positive number, $C_{x, t}^{s, s / 2}(\bar{Q})$ and $C^{s}(\bar{\Omega})$ denote the Hölder spaces as defined in [4]. We consider:

Problem 1 : The first type boundary value problem for one dimensional heat equation

$$
\begin{aligned}
& L u=f(x, t) \text { on } Q_{T}, \\
& u(x, 0)=u_{0}(x) \text { on } \gamma_{2}, \\
& u(0, t)=u_{1}(t) \text { on } \gamma_{1}, u(b, t)=u_{2}(t) \text { on } \gamma_{3},
\end{aligned}
$$

Where $L \equiv \frac{\partial}{\partial t}-a \frac{\partial^{2}}{\partial x^{2}}, a$ is positive constant and

$u_{0}(x) \in C^{8+\alpha}(\bar{\Omega}), f(x, t) \in C_{x, t}^{6+\alpha, 3+\frac{\alpha}{2}}\left(\bar{Q}_{T}\right)$ and $u_{j}(t) \in C^{4+\frac{\alpha}{2}}\left(\bar{\sigma}_{T}\right), j=1,2$,

also the conjugation conditions

$$
\begin{aligned}
& u^{(q)}(0)=u_{1}^{(q)}(0) \\
& u^{(q)}(b)=u_{2}^{(q)}(0)
\end{aligned}
$$

of order $q=0,1,2,3,4$ between the initial and boundary conditions are satisfied (see [4]). Here, 


$$
\begin{aligned}
& u^{(0)}(x)=u_{0}(x), u^{(q)}(x)=\left.\partial_{t}^{q} u(x, t)\right|_{t=0}, q=1,2, \ldots, \\
& u_{1}^{(0)}(0)=\left.u_{1}(t)\right|_{t=0}, u_{2}^{(0)}(0)=\left.u_{2}(t)\right|_{t=0} \\
& u_{1}^{(q)}(0)=\left.D_{t}^{q} u_{1}(t)\right|_{t=0}, u_{2}^{(q)}(0)=\left.D_{t}^{q} u_{2}(t)\right|_{t=0}, q=1,2, \ldots
\end{aligned}
$$

$\partial_{t}^{q} u(x, t)=\frac{\partial^{q} u}{\partial t^{q}}, D_{t}^{q} u_{i}(t)=\frac{d^{q} u_{i}}{d t^{q}} \quad i=1,2$ and $u^{(q)}(x), q=1,2,3,4$ in (7) are defined as

$$
\begin{aligned}
& u^{(1)}(x)=a D_{x}^{2} u_{0}(x)+f(x, 0), \\
& u^{(q)}(x)=a \partial_{x}^{2} u^{(q-1)}(x)+f^{(q-1)}(x),
\end{aligned}
$$

where, $f^{(0)}(x)=f(x, 0)$ and $f^{(q)}(x)=\left.\partial_{t}^{q} f(x, t)\right|_{t=0}$. Let $w=\partial_{t} u$ and $\phi=\partial_{x}^{2} u$. The following boundary value problems are considered [5]

\section{Problem 2}

\section{Problem 3}

$$
\begin{aligned}
& L w=\partial_{t} f(x, t)=F(x, t) \text { on } Q_{T}, \\
& w(x, 0)=u^{(1)}(x)=w_{0}(x) \text { on } \gamma_{2}, \\
& w(0, t)=D_{t} u_{1}(t)=w_{1}(t) \text { on } \gamma_{1}, \\
& w(b, t)=D_{t} u_{2}(t)=w_{2}(t) \text { on } \gamma_{3},
\end{aligned}
$$

We represent the set of nodes on $\gamma_{i}, i=1,2,3$ by

$$
\begin{gathered}
\omega_{0, \tau}=\left\{\left(0, t_{j}\right): t_{j}=j \tau, \tau=\frac{T}{M}, j=0, \ldots, M\right\}, \\
\bar{\omega}_{h, 0}=\left\{\left(x_{m}, 0\right): x_{m}=m h, h=\frac{b}{N}, m=0, \ldots, N\right\}, \\
\omega_{b, \tau}=\left\{\left(b, t_{j}\right): t_{j}=j \tau, \tau=\frac{T}{M}, j=0, \ldots, M\right\},
\end{gathered}
$$

respectively. To realize the numerical solution of the Problem 2, and Problem 3 we use implicit four-point difference scheme [6]. Let $\tilde{w}$ denote the solution of

$$
\begin{aligned}
& \widetilde{w}_{t, m}^{h, \tau}=a \Lambda \widetilde{w}_{m}^{h, \tau}+\varphi_{\tilde{w}, m} \text { on } \quad \omega_{h, \tau}, \\
& \widetilde{w}_{m}^{0}=\widetilde{w}\left(x_{m}, 0\right)=w_{0}\left(x_{m}\right) \text { on } \quad \bar{\omega}_{h, 0}, \\
& \widetilde{w}_{0}^{j}=\widetilde{w}\left(0, t_{j}\right)=w_{1}\left(t_{j}\right) \text { on } \quad \omega_{0, \tau}, \widetilde{w}_{N}^{j}=\tilde{w}\left(b, t_{j}\right)=w_{2}\left(t_{j}\right) \text { on } \omega_{b, \tau},
\end{aligned}
$$

where,

$$
\tilde{w}_{t, m}^{h, \tau}=\frac{1}{\tau}\left(\tilde{w}_{m}^{j+1}-\tilde{w}_{m}^{j}\right),
$$




$$
\begin{aligned}
\Lambda \tilde{w}_{m}^{h, \tau} & =\frac{1}{h^{2}}\left(\tilde{w}_{m+1}^{j+1}-2 \tilde{w}_{m}^{j+1}+\tilde{w}_{m-1}^{j+1}\right), \\
\varphi_{\tilde{w}, m} & =F\left(x_{m}, t_{j+1}\right)=\partial_{t} f\left(x_{m}, t_{j+1}\right) .
\end{aligned}
$$

For the approximate solution of Problem 3 we give the next four point difference problem and denote the solution by $\tilde{\phi}$

$$
\begin{aligned}
& \widetilde{\phi}_{t, m}^{h, \tau}=a \Lambda \tilde{\phi}_{m}^{h, \tau}+\varphi_{\tilde{\phi}, m} \text { on } \omega_{h, \tau}, \\
& \tilde{\phi}_{m}^{0}=\tilde{\phi}\left(x_{m}, 0\right)=\phi_{0}\left(x_{m}\right) \text { on } \bar{\omega}_{h, 0} \\
& \widetilde{\phi}_{0}^{j}=\tilde{\phi}\left(0, t_{j}\right)=\phi_{1}\left(t_{j}\right) \text { on } \omega_{0, \tau}, \widetilde{\phi}_{N}^{j}=\tilde{\phi}\left(b, t_{j}\right)=\phi_{2}\left(t_{j}\right) \text { on } \omega_{b, \tau},
\end{aligned}
$$

where, $\tilde{\phi}_{\bar{t}, m}^{h, \tau}, \Lambda \tilde{\phi}_{m}^{h, \tau}$ are formulae analogous to (23) and (24) respectively, and $\varphi_{\tilde{\phi}, m}=G\left(x_{m}, t_{j+1}\right)=\left.\partial_{x}^{2} f\right|_{\left(x_{m}, t_{j+1}\right)}$. We denote $q_{i}=\partial_{t}^{2} u$ and $p_{i}=\partial_{x} \partial_{t} u$ on $\gamma_{i}, i=1,2,3$, and construct the boundary value Problem 4 [5] for $z=\partial_{t}^{2} u$, and Problem 5 for $y=\partial_{x} \partial_{t} u$.

\section{Problem 4}

$$
\begin{aligned}
& L z=\partial_{t}^{2} f(x, t) \text { on } Q_{T}, \\
& z(x, 0)=q_{2} \text { on } \gamma_{2}, \\
& z(0, t)=q_{1} \text { on } \gamma_{1}, z(b, t)=q_{3} \text { on } \gamma_{3},
\end{aligned}
$$

where, $f(x, t)$ is the heat source function in (1). We take

$$
\begin{gathered}
q_{1 \tau}=D_{t}^{2} u_{1}(t)=D_{t} w_{1}(t) \text { on } \omega_{0, \tau} \\
q_{2 \tau}(\tilde{w})=\frac{1}{\tau}\left(\widetilde{w}(x, \tau)-w_{0}(x)\right) \text { on } \bar{\omega}_{h, 0}, \\
q_{3 \tau}=D_{t}^{2} u_{2}(t)=D_{t} w_{2}(t) \text { on } \omega_{b, \tau},
\end{gathered}
$$

where, $w_{0}(x)=u^{(1)}(x)$ is as defined in (10) and $\boldsymbol{u}_{1}(t), \boldsymbol{u}_{2}(t)$ are given in (3). We propose the next difference problem for the numerical solution of Problem 4

$$
\begin{aligned}
& \widetilde{z}_{\bar{t}, m}^{h, \tau}=a \Lambda \widetilde{z}_{m}^{h, \tau}+\varphi_{\tilde{z}, m} \text { on } \omega_{h, \tau}, \\
& \widetilde{z}_{m}^{0}=q_{2 \tau}(\tilde{w}) \text { on } \bar{\omega}_{h, 0}, \\
& \widetilde{z}_{0}^{j}=q_{1 \tau} \text { on } \omega_{0, \tau}, \widetilde{z}_{N}^{j}=q_{3 \tau} \text { on } \omega_{b, \tau},
\end{aligned}
$$

where $q_{i \tau}, i=1,2,3$ are defined by (32)-(34) and $\varphi_{\tilde{z}, m}=\left.\partial_{t}^{2} f\right|_{\left(x_{m}, t_{j+1}\right)}$.

\section{Problem 5}

$$
\begin{aligned}
& L y=\partial_{x} \partial_{t} f(x, t) \text { on } Q_{T}, \\
& y(x, 0)=p_{2} \text { on } \gamma_{2}, \\
& y(0, t)=p_{1} \text { on } \gamma_{1}, y(b, t)=p_{3} \text { on } \gamma_{3},
\end{aligned}
$$

where, $f(x, t)$ is the given function in (1). We take 


$$
\begin{gathered}
p_{1 h}(\widetilde{w})=\frac{1}{2 h}\left(-3 w_{1}(t)+4 \widetilde{w}(h, t)-\widetilde{w}(2 h, t)\right) \text { on } \omega_{0, \tau} \\
p_{2 h}=D_{x} w_{0}(x) \text { on } \bar{\omega}_{h, 0} \\
p_{3 h}(\widetilde{w})=\frac{1}{2 h}\left(3 w_{2}(t)-4 \widetilde{w}(b-h, t)+\widetilde{w}(b-2 h, t)\right) \text { on } \omega_{b, \tau}
\end{gathered}
$$

where, $w_{0}(x)=u^{(1)}(x)$ and $w_{1}=D_{t} u_{1}(t), w_{2}=D_{t} u_{2}(t)$.

For the numerical solution of Problem 5 we propose the next difference problem

$$
\begin{aligned}
& \tilde{y}_{\bar{t}, m}^{h, \tau}=a \Lambda \tilde{y}_{m}^{h, \tau}+\varphi_{\tilde{y}, m} \text { on } \omega_{h, \tau}, \\
& \tilde{y}_{m}^{0}=\tilde{y}\left(x_{m}, 0\right)=p_{2 h} \text { on } \bar{\omega}_{h, 0}, \\
& \tilde{y}_{0}^{j}=\tilde{y}\left(0, t_{j}\right)=p_{1 h}(\widetilde{w}) \text { on } \omega_{0, \tau}, \tilde{y}_{N}^{j}=\tilde{y}\left(b, t_{j}\right)=p_{3 h}(\tilde{w}) \text { on } \omega_{b, \tau},
\end{aligned}
$$

and denote the solution by $\tilde{y}$. Here, $p_{i h}$ are defined by (41)-(43) and $\tilde{y}_{\bar{t}, m}^{h, \tau}, \Lambda \tilde{y}_{m}^{h, \tau}$ are formulae analogous to (23) and (24) respectively, also $\varphi_{\tilde{y}, m}=\left.\partial_{x} \partial_{t} f\right|_{\left(x_{m}, t_{j+1}\right)}$.

Theorem : The solution $\tilde{z}$ of the finite difference problem (35)-(37) satisfies

$$
\max _{\bar{\omega}_{h, \tau}}|\tilde{z}-z| \leq c_{1}\left(h^{2}+\tau\right)
$$

and the solution $\tilde{y}$ of the finite difference problem (44)-(46) satisfies

$$
\max _{\bar{\omega}_{h, \tau}}|\tilde{y}-y| \leq c_{2}\left(h^{2}+\tau\right)
$$

where, $z=\partial_{t}^{2} u$ is the exact solution of Problem 4, and $y=\partial_{x} \partial_{t} u$ is the exact solution of Problem 5 also, $c_{1}, c_{2}$ are constants independent from $h$ and $\tau$.

Remark : The extended theoretical analysis and numerical examples will be presented in the full research article.

\section{References}

1. J.Douglas, H.H. Rachford, Trans. Amer. Math. Soc. 82, No.2. 421-439, (1956)

2. H.G. Landau, Quart. Appl, Math. 8, 81-94 (1950)

3. M. Lotkin, Quart. Appl, Math. 18(1), 79-85 (1960).

4. O.A. Ladyzenskaja, V.A. Solonnikov, N.N. Ural'ceva, Translation of Mathematical Monographs, American Mathematical Society, USA, (1967).

5. S.C. Buranay, L.A. Farinola, Proceedings of Fourth International Conference on Analysis and Applied Mathematics (ICAAM 2018), AIP publishing (accepted for publication).

6. A.A. Samarskii, Marcel Dekker, Inc. New York (2001). 